\title{
USE OF CLASSICAL VARIATIONAL PRINCIPLES TO DETERMINE BOUNDS FOR THE EFFECTIVE BULK MODULUS IN HETEROGENEOUS MEDIA*
}

\author{
BY \\ M. BERAN (University of Pennsylvania) AND J. MOLYNEUX** (New York University)
}

\begin{abstract}
Bounds are here derived for the effective bulk modulus in heterogeneous media, denoted by $k^{*}$, using the two standard variational principles of elasticity. As trial functions for the stress and strain fields we use perturbation expansions that have been modified by the inclusion of a set of multiplicative constants. The first order perturbation effect is explicitly calculated and bounds for $k^{*}$ are derived in terms of the correlation functions $\left\langle\mu^{\prime}(0) k^{\prime}(\mathbf{r}) k^{\prime}(\mathbf{s})\right\rangle$ and $\left\langle\left[k^{\prime}(\mathbf{r}) k^{\prime}(\mathbf{s}) / \mu(0)\right]\right\rangle$ where $\mu^{\prime}$ and $k^{\prime}$ are the fluctuating parts of the shear modulus $\mu$ and the bulk modulus, $k$, respectively. Explicit calculations are given for two phase media when $\mu^{\prime}(\mathbf{x})=0$ and when the media are symmetric in the two phases. Results are also included for the dielectric problem when the media are composed of two symmetric phases.
\end{abstract}

1. Introduction. The principles of minimum potential energy and minimum complementary energy have been used by many authors (See Hashin [1] for appropriate references) to show that the effective bulk modulus of a heterogeneous material, denoted by $k^{*}$, is bounded by the relation

$$
\frac{1}{\left\langle\frac{1}{k}\right\rangle} \leq k^{*} \leq\langle k\rangle
$$

where the brackets denote an average value. $\dagger$ For a medium in which $k$ is constrained to lie between two values $k_{1}$ and $k_{2}$ (such as a two phase medium) better bounds may be derived for $k^{*}$ (Hashin and Shtrikman [2]). If, however, $k$ may take on any value between 0 and $\infty$ (1) appears to be all one can say about $k^{*}$ if nothing more is known than the fraction of the medium that has a particular value $k$. The purpose of this paper is to show how the bounds on $k^{*}$ may be narrowed if more information is known about the geometry of the heterogeneous medium.

Most heterogeneous materials may be conveniently described in a statistical sense and we consider in this paper only media that may be so represented. For the purpose of calculating the effective bulk modulus we assume that we have an ensemble of media that are statistically homogeneous and isotropic. The usual volume averages of a single large sample are here replaced by an ensemble average (assuming an ergodic hypothesis) and the ordinary variational principles of elasticity which contain volume integrals are replaced by ensemble averages. The media are generally characterized by the entire sequence of correlation functions

$$
R_{k_{m} \mu_{n}} \equiv\left\langle k\left(\mathbf{x}_{1}\right) \cdots k\left(\mathbf{x}_{m}\right) \mu\left(\mathbf{x}_{m+1}\right) \cdots \mu\left(\mathbf{x}_{m+n}\right)\right\rangle
$$

${ }^{*}$ Received May 10, 1965; revised manuscript received December 3, 1965. The work was partially supported by the U. S. Army Research Office, Durham, North Carolina. Grant DA-ARO-D31-124G-331, and National Science Foundation, Grant No. GP-3668.

**Present address, University of Rochester.

tThe type of average used in this paper is discussed below. 
or some equivalent information (the brackets denote an ensemble average). For example, to determine the bounds in (1) we must know $R_{k_{m} \mu_{\mathrm{o}}}$ for $\mathbf{x}_{1}=\mathbf{x}_{2}=\cdots \mathbf{x}_{m}$. This is denoted by $\left\langle k^{m}\right\rangle$. Under usual physical conditions $P(k) d k$, the probability of finding a particular value of $k$ at any point in the medium, is determined from knowledge of all the $\left\langle k^{m}\right\rangle$ and from $P(k)$ we may find $\langle k\rangle$ and $\langle 1 / k\rangle$. To get better bounds for $k^{*}$ than those given in (1) we must have a knowledge of higher order moments such as

$$
\begin{aligned}
& \left\langle k\left(\mathbf{x}_{1}\right) k\left(\mathbf{x}_{2}\right)\right\rangle,\left\langle k\left(\mathbf{x}_{1}\right) k\left(\mathbf{x}_{2}\right) k\left(\mathbf{x}_{3}\right)\right\rangle, \\
& \left\langle\mu\left(\mathbf{x}_{1}\right) k\left(\mathbf{x}_{2}\right)\right\rangle,\left\langle\mu\left(\mathbf{x}_{1}\right) k\left(\mathbf{x}_{2}\right) k\left(\mathbf{x}_{3}\right)\right\rangle, \cdots
\end{aligned}
$$

In this paper we shall show how the information given by higher order moments may be incorporated into the classical variational principles of elasticity by appropriate choice of trial function. This was recently done for the dielectric problem by Beran [3]. A statistical theory of heterogeneous materials was presented by Molyneux and Beran [4] for media with small perturbations in elastic moduli. In a paper on media with variable permittivity Beran and Molyneux [5] showed how to formulate the higher order equations using perturbation theory. Here we shall use the formalism developed in these papers to chose trial functions that give bounds for $k^{*}$ in terms of the correlation functions describing the geometry of the media.

In section two we shall state the classical variational principles and then give a restatement of these principles in terms of ensemble averages. In section three we shall discuss the trial functions which will be used in the variational principles and then derive bounds for $k$ including first order effects. The zero order trial function simply gives (1). We conclude the paper with the explicit determination of $k^{*}$ for media in which the fluctuations are small, those in which $\mu^{\prime}(\mathbf{x})=0$ where $\mu^{\prime}(\mathbf{x})$ is the fluctuation in shear modulus and for two phase media that are symmetric in the two phases. This latter result will be compared to the results of Hashin and Shtrikman [2]. We will also include some results for two phase symmetric media in connection with the dielectric problem since they have not been published elsewhere.

2. Variational Principles. Principle of Minimum Potential Energy: Let an isotropic elastic body occupying the volume $V$ be in equilibrium under the action of specified surface displacements. The principle states that the expression

$$
W=\frac{1}{2 V} \int_{V}\left(k \theta^{2}+2 \mu \pi_{i i} \pi_{i j}\right) d \mathbf{x}
$$

where $\pi_{i j}=e_{i j}-\frac{1}{3} \theta \delta_{i j}, \theta=e_{i i}, k$ and $\mu$ are the bulk and shear modulus respectively and the strain tensor $e_{i j}$ is subject to the subsidiary condition

$$
e_{i j}=\frac{1}{2}\left(\frac{\partial u_{i}}{\partial x_{i}}+\frac{\partial u_{i}}{\partial x_{i}}\right),
$$

is stationary and assumes its minimum value for strains satisfying

$$
\frac{\partial}{\partial x_{i}}\left[k \theta \delta_{i i}+2 \mu \pi_{i j}\right]=0 .
$$

The last equation is simply $\partial \tau_{i j} / \partial x_{i}=0$ where $\tau_{i j}$ is the stress tensor. We, of course, impose the requirement that the variation of $u_{i}$ is zero on the boundary. (See Sokolnikoff [6] for a proof of this theorem and for a discussion of the principle of minimum complementary energy). 
For purposes of calculating $k^{*}$ we assume an ergodic hypothesis and replace the expression for $W$ by the expression

$$
W_{E}=\frac{1}{2}\left\langle\left(k \theta^{2}+2 \mu \pi_{i j} \pi_{i j}\right)\right\rangle,
$$

where the brackets denote an ensemble average. We choose $\left\langle u_{i}\right\rangle=-B x_{i}$ and assume that $W_{E}$ is independent of $\mathbf{x}$. For this replacement we require that the volume $V$ be very very large compared to characteristic distances over which $k$ and $\mu$ vary. In fact in this ensemble formulation we take $V$ to be infinite. Further we require that the variations in any part of $V$ be homogeneous and isotropic when considered in a statistical sense. The subsidiary condition relating $e_{i j}$ and $u_{i}$ remains the same as in the volume integral. The only extra condition we require is a condition to replace the requirement $\delta u_{i}=0$ on $S$. The condition we now use is that $\delta u_{i}$ must have statistics that are homogeneous and $\left\langle\delta u_{i}\right\rangle=0$. From this it follows that $\left\langle u_{i}\right\rangle$ must be the same for all admissible solutions. An admissible solution $u_{i A}$ is thus a solution which has homogeneous statistics and for which $\left\langle u_{i A}\right\rangle$ is equal to the mean value of the true solution. For an admissible solution $u_{i \mathrm{~A}}$

$$
W_{E} \leq \frac{1}{2}\left\langle\left(k \theta_{A}^{2}+2 \mu \pi_{i j A} \pi_{i j A}\right)\right\rangle .
$$

We define $k^{*}$ by the following relation

$$
\frac{1}{2} k^{*}\langle\theta\rangle^{2}=W_{E} .
$$

Thus

$$
k^{*} \leq \frac{1}{\langle\theta\rangle^{2}}\left\langle\left(k \theta_{A}^{2}+2 \mu \pi_{i j A} \pi_{i j A}\right)\right\rangle .
$$

Principle of Minimum Complementary Energy. The integral

$$
W=\frac{1}{2 V} \int_{V}\left[\frac{1}{9 k} \psi^{2}+\frac{1}{2 \mu} \beta_{i j} \beta_{i i}\right] d \mathbf{x}
$$

where $\beta_{i i}=\tau_{i i}-\frac{1}{3} \psi \delta_{i i}, \psi=\tau_{i i}$, and the stress tensor $\tau_{i i}$ is subject to the subsidiary conditions

$$
\tau_{i, i}=0 ; \quad \tau_{i j}=\tau_{i i}
$$

is stationary and a minimum for stresses derivable from a displacement field:

$$
\frac{4}{6 \mu k}\left(\frac{2}{3} \mu-k\right) \tau_{l l} \delta_{i i}+\frac{\tau_{i j}}{2 \mu}=e_{i j}=\frac{1}{2}\left(\frac{\partial u_{i}}{\partial x_{i}}+\frac{\partial u_{i}}{\partial x_{i}}\right) .
$$

We impose the requirement that the variations of the tractions on $S$ are zero.

Proceeding as we did in the minimum potential energy case we replace $W$ by $W_{\boldsymbol{E}}$ where

$$
W_{B}=\frac{1}{2}\left\langle\left[\frac{1}{9 k} \psi^{2}+\frac{1}{2 \mu} \beta_{i j} \beta_{i j}\right]\right\rangle .
$$

Here we require that $\tau_{i j A}$ have homogeneous statistics and that $\left\langle\tau_{i j A}\right\rangle$ be equal to the mean value of the true solution.

For any admissible solution $\tau_{i j A}$

$$
W_{E} \leq \frac{1}{2}\left\langle\left(\frac{1}{9 k} \psi_{A}^{2}+\frac{1}{2 \mu} \beta_{i j A} \beta_{i ; A}\right)\right\rangle .
$$


Since $\psi \equiv \tau_{i i}=3 k e_{i i} \equiv 3 k \theta$

$$
W_{E}=\frac{1}{18} k^{*}\left\langle\left[\frac{\psi}{k}\right]\right\rangle^{2} .
$$

To find a lower bound for $k^{*}$ we consider the special case when $\left\langle\beta_{i j}\right\rangle=0$; that is the average stress field corresponds to a hydrostatic pressure field. In this case it may be shown that

$$
W_{B}=\frac{1}{2}\left\langle\frac{\psi}{9 k}\right\rangle\langle\psi\rangle, \quad\left(\left\langle\beta_{i i}\right\rangle=0\right) .
$$

Combination of (10), (11), and (12) yields

$$
\frac{1}{9} \frac{\langle\psi\rangle^{2}}{k^{*}} \leq\left\langle\frac{1}{9 k} \psi_{A}^{2}+\frac{1}{2 \mu} \beta_{i ; A} \beta_{i j A}\right\rangle \quad\left(\left\langle\beta_{i j}\right\rangle=0\right)
$$

or

$$
k^{*} \geq \frac{\frac{1}{9}\langle\psi\rangle^{2}}{\left\langle\frac{1}{9 k} \psi_{A}^{2}\right\rangle+\left\langle\frac{1}{2 \mu} \beta_{i, A} \beta_{i j A}\right\rangle} \quad\left(\left\langle\beta_{i j}\right\rangle=0\right) .
$$

3. Trail functions for the two principles. Upper bound obtained from the Principle of Minimum Potential Energy. We will take as a trial function a modification of the perturbation series that one would obtain for $e_{i}$ if one expanded $e_{i j}$ in a series

$$
e_{i j}(\mathbf{x})=\left\langle e_{i i}\right\rangle+e_{i j}^{(1)}(\mathbf{x})+e_{i i}^{(2)}(\mathbf{x})+\cdots,
$$

where we let $e_{i j}^{(1)}(\mathbf{x})$ be of order $k^{\prime} /\langle k\rangle$, ( $k^{\prime}$ is the fluctuation in bulk modulus about the mean) $e_{i j}^{(2)}$ be of order $k^{\prime 2} /\langle k\rangle^{2}$ and so on, and we suppose that $k^{\prime} /\langle k\rangle \ll 1$. The lowest order approximation is obtained by simply taking $e_{i j}(\mathbf{x})=\left\langle e_{i j}\right\rangle$. To include the first order effect we would take

$$
e_{i j}(\mathbf{x})=\left\langle e_{i j}\right\rangle+e_{i j}^{(1)}(\mathbf{x}) .
$$

The first order problem has been considered in detail by Molyneux and Beran [4] and we shall later make use of their results.

We assume that if the infinite series represented in (15) were used in (7) as an admissible solution we would get an equality. To make use of a portion of this series we write

$$
{ }_{N} e_{i j A}(\mathbf{x})=\left\langle e_{i j}\right\rangle+\alpha_{1} e_{i j}^{(1)}(\mathbf{x})+\alpha_{2} e_{i j}^{(2)}(\mathbf{x})+\cdots+\alpha_{N} e_{i j}^{(N)}(\mathbf{x}),
$$

where the $\alpha_{i}$ are to be chosen to obtain the best results when these finite number of terms are used as an admissible solution. If an infinite number of terms were used we would have $\alpha_{i}=1$. In this paper we shall consider in detail a trial function of the form

$$
e_{i j A}(\mathbf{x})=\left\langle e_{i j}\right\rangle+\alpha_{1} e_{i i}^{(1)}(\mathbf{x}) .
$$

The simplest trial function to choose would have been

$$
e_{i j A}(x)=\left\langle e_{i i}\right\rangle,
$$

with $\left\langle\pi_{i j}\right\rangle=0$. 
Substituting (18) into (7) we obtain the upper bound given in (1):

$$
k^{*} \leq\langle k\rangle \text {. }
$$

The trial function given in (17) will yield an upper bound for $k^{*}$ in terms of a third order correlation function.

Substitution of (17) into (7) yields

$k^{*} \leq \frac{1}{\langle\theta\rangle^{2}}\left\{\langle k\rangle\langle\theta\rangle^{2}+\alpha_{1}^{2}\langle k\rangle\left\langle\theta^{(1) 2}\right\rangle+2 \alpha_{1}\left\langle k^{\prime} \theta^{(1)}\right\rangle\langle\theta\rangle+\alpha_{1}^{2}\left\langle k^{\prime} \theta^{(1) 2}\right\rangle\right.$

$$
\left.+2\left[\alpha_{1}^{2}\langle\mu\rangle\left\langle\pi_{i j}^{(1)} \pi_{i j}^{(1)}\right\rangle+\alpha_{1}^{2}\left\langle\mu^{\prime} \pi_{i j}^{(1)} \pi_{i j}^{(1)}\right\rangle\right]\right\}
$$

where

$$
\begin{gathered}
\theta^{(1)}=e_{i i}^{(1)}, \\
\pi_{i j}^{(1)}=e_{i j}^{(1)}-\frac{1}{3} \theta^{(1)} \delta_{i j},
\end{gathered}
$$

and $\alpha_{1}$ is to be chosen to minimize the right-hand side of (20).

Straight forward manipulation with the equation

$$
\frac{\partial \tau_{i j}}{\partial x_{i}}=\frac{\partial}{\partial x_{i}}\left[k \theta \delta_{i i}+2 \mu \pi_{i i}\right]=0,
$$

(Molyneux and Beran [4]) yields

$$
\begin{gathered}
\nabla^{2} \theta^{(1)}=-\frac{\langle\theta\rangle}{\langle\lambda\rangle+2\langle\mu\rangle} \nabla^{2} k^{\prime} \\
\langle\mu\rangle \nabla^{2} \pi_{i i}^{(1)}=(\langle\lambda\rangle+\langle\mu\rangle\rangle\langle\theta\rangle_{, i}\left[\frac{1}{3} \frac{\langle\mu\rangle\langle\theta\rangle}{(\langle\lambda\rangle+2\langle\mu\rangle)}\right] \delta_{i i} \nabla^{2} k^{\prime}-\langle\theta\rangle k^{\prime},_{i j},
\end{gathered}
$$

where $\lambda$ is one of the Lamé constants $\left(k=\lambda+\frac{2}{3} \mu\right)$.

From these equations we find

$$
\theta^{(1)}(\mathbf{x})=\frac{1}{4 \pi} \int_{V} \frac{\langle\theta\rangle}{(\langle\lambda\rangle+2\langle\mu\rangle)} \frac{\partial^{2} k^{\prime}(\mathbf{n})}{\partial \eta_{i} \partial \eta_{i}} \frac{1}{r(\mathbf{n}, \mathbf{x})} d \mathbf{n},
$$

where $r(\mathbf{n}, \mathbf{x})=\sqrt{\left(\eta_{x}-x\right)^{2}+\left(\eta_{y}-y\right)^{2}+\left(\eta_{z}-z\right)^{2}}$ and the integral is taken over the entire space.* Also,

$$
\pi_{i j}^{(1)}(\mathbf{x})=\frac{\langle\theta\rangle}{(\langle\lambda\rangle+2\langle\mu\rangle)}\left[\frac{1}{3} k^{\prime} \delta_{i i}+\frac{1}{4 \pi} \int_{V} \frac{\partial^{2} k^{\prime}(\mathbf{n})}{\partial \eta_{i}} \frac{1}{\partial \eta_{i}} \frac{1}{r(\mathbf{n}, \mathbf{x})} d \mathbf{n}\right] .
$$

From (24) and (25) we may find the quantities needed to evaluate (20);

$$
\left\langle\theta^{(1) 2}\right\rangle,\left\langle k^{\prime} \theta^{(1)}\right\rangle,\left\langle k^{\prime} \theta^{(1) 2}\right\rangle,\left\langle\pi_{i j}^{(1)} \pi_{i j}^{(1)}\right\rangle,\left\langle\mu^{\prime} \pi_{i i}^{(1)} \pi_{i i}^{(1)}\right\rangle .
$$

To find $\left\langle k^{\prime} \theta^{(1)}\right\rangle$ for example multiply both sides of $(24)$ by $k^{\prime}(\mathbf{x})$ and take the ensemble average of the equation. Assuming that the integration commutes with the ensemble averaging we have**

*Strictly speaking this is incorrect since the integral is divergent. 'To give a more proper formalism we must take $k^{\prime}$ to be zero outside some finite volume and then subsequently allow the volume to become infinite. In the interests of simplicity we shall omit this limiting procedure since equations like Eq. 26 are the same in both cases.

**To justify this we have to go through the limiting procedure indicated in the above footnote. 


$$
\left\langle k^{\prime} \theta^{(1)}\right\rangle=\frac{1}{4 \pi} \int \frac{\langle\theta\rangle}{\langle\lambda\rangle+2\langle\mu\rangle} \frac{\partial^{2}\left\langle k^{\prime}(\mathbf{x}) k^{\prime}(\mathbf{n})\right\rangle}{\partial \eta_{i} \partial \eta_{i}} \frac{1}{r(\mathbf{n}, \mathbf{x})} d \mathbf{n} .
$$

Since the media are statistically homogeneous and isotropic

$$
\left\langle k^{\prime}(\mathbf{x}) k^{\prime}(\mathfrak{n})\right\rangle \equiv K^{\prime}(|\mathbf{x}-\mathbf{n}|) .
$$

Transforming to the variable $\mathbf{p}=\mathbf{x}-\mathfrak{n}$ we have

$$
\left\langle k^{\prime} \theta^{(1)}\right\rangle=\frac{1}{4 \pi} \frac{\langle\theta\rangle}{\langle\lambda\rangle+2(\mu)} \int_{V} \frac{\partial^{2} K^{\prime}(\mathbf{p})}{\partial p_{i} \partial p_{i}} \frac{1}{p} d \mathbf{p}
$$

where $p^{2}=p_{i} p_{i}$. Since

$$
\frac{\partial^{2} K^{\prime}(p)}{\partial p_{i} \partial p_{i}}=\frac{1}{p^{2}} \frac{\partial}{\partial p} p^{2} \frac{\partial K^{\prime}}{\partial p}
$$

this integral may be readily integrated over the spherical coordinates $\theta$ and $\phi$ to find

$$
\left\langle k^{\prime} \theta^{(1)}\right\rangle=\frac{\langle\theta\rangle}{\langle\lambda\rangle+2\langle\mu\rangle} \int_{0}^{\infty}\left(\frac{\partial}{\partial p} p^{2} \frac{\partial K^{\prime}}{\partial p}\right) \frac{d p}{p},
$$

and

$$
\left\langle k^{\prime} \theta^{(1)}\right\rangle=-\frac{\langle\theta\rangle}{\langle\lambda\rangle+2\langle\mu\rangle}\left\langle k^{\prime 2}\right\rangle,
$$

assuming that $p \partial K^{\prime} / \partial p \rightarrow 0$ as $p \rightarrow \infty$. Note also that $K^{\prime}(0)=\left\langle k^{\prime 2}\right\rangle$.

In a similar manner we find

$$
\begin{gathered}
\left\langle\theta^{(1) 2}\right\rangle=\frac{\langle\theta\rangle^{2}}{(\langle\lambda\rangle+2\langle\mu\rangle)}\left\langle k^{\prime 2}\right\rangle, \\
\left\langle k^{\prime} \theta^{(1) 2}\right\rangle=\frac{\langle\theta\rangle^{2}}{(\langle\lambda\rangle+2\langle\mu\rangle)^{2}}\left\langle k^{\prime 3}\right\rangle, \\
\left\langle\pi_{i i}^{(1)} \pi_{i i}^{(1)}\right\rangle=\frac{2}{3} \frac{\langle\theta\rangle^{2}}{(\langle\lambda\rangle+2\langle\mu\rangle)^{2}}\left\langle k^{\prime 2}\right\rangle, \\
\left\langle\mu^{\prime} \pi_{i i}^{(1)} \pi_{i i}^{(1)}\right\rangle=\frac{\langle\theta\rangle^{2}}{(\langle\lambda\rangle+2\langle\mu\rangle)^{2}}\left[-\frac{1}{3}\left\langle\mu^{\prime} k^{\prime 2}\right\rangle+J\right],
\end{gathered}
$$

where

$$
J=\frac{1}{16 \pi^{2}} \int_{\xi} \int_{\mathbf{n}} \frac{\partial^{2}}{\partial \xi_{i} \partial \xi_{i}} \frac{\partial^{2}}{\partial \eta_{i} \partial \eta_{i}}\left\langle\mu^{\prime}(0) k^{\prime}(\xi) k^{\prime}(\mathfrak{n})\right\rangle \frac{1}{|\xi||\mathbf{n}|} d \xi d \mathfrak{n} .
$$

Using (28)-(33) in (20) we find

$$
\begin{aligned}
& k^{*} \leq\langle k\rangle-\frac{2 \alpha\left\langle k^{\prime 2}\right\rangle}{(\langle\lambda\rangle+\langle\mu\rangle)}+\frac{\alpha^{2}}{(\langle\lambda\rangle+2\langle\mu\rangle)^{2}} \\
& \cdot\left[\langle k\rangle\left\langle k^{\prime 2}\right\rangle+\left\langle k^{\prime 3}\right\rangle+\frac{4}{3}\langle\mu\rangle\left\langle k^{\prime 2}\right\rangle-\frac{2}{3}\left\langle\mu^{\prime} k^{\prime 2}\right\rangle+2 J\right] .
\end{aligned}
$$

We now want to minimize the right hand side of (34) with respect to $\alpha$. We note first that (34) is of the form

$$
k^{*} \leq\langle k\rangle+2 B \alpha+A \alpha^{2}
$$


where from (20) and (29) we have

$$
B=\frac{\left\langle k^{\prime} \theta^{(1)}\right\rangle}{\langle\theta\rangle}=-\frac{1}{\langle\lambda\rangle+2\langle\mu\rangle}\left\langle k^{\prime 2}\right\rangle \leq 0
$$

and from (20)

$$
A=\frac{1}{\langle\theta\rangle^{2}}\left[\left\langle k \theta^{(1)^{2}}\right\rangle+\left\langle 2 \mu \pi_{i j}^{\prime} \pi_{i j}^{\prime}\right\rangle\right] \geq 0 .
$$

Finding the stationary value of (34) with respect to $\alpha$ and noting that $A \geq 0$ implies

$$
A \alpha_{S}^{2}+2 B \alpha_{S}=-\frac{B^{2}}{A} \leq 0,
$$

we observe that (35) will yield at least as good an upper bound as the one given in (1). Substituting $\alpha_{S}$ into (34) yields finally

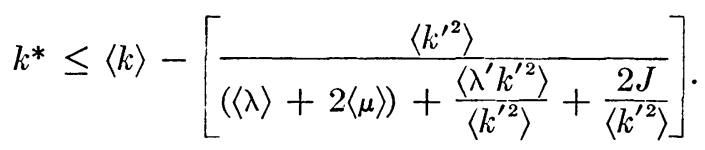

Lower Bound obtained from the Principle of Complementary Energy. It is more difficult to obtain the perturbation equations for the stress boundary value problem. To solve this problem we will construct the equations to be satisfied by our trial functions in a somewhat artificial manner. As in the displacement case we seek in general a trial function of the form

$$
{ }_{N} \tau_{i j A}=\left\langle\tau_{i j}\right\rangle+\eta_{1} \tau_{i j}^{(1)}(\mathbf{x})+\eta_{2} \tau_{i j}^{(2)}(\mathbf{x})+\cdots+\eta_{N} \tau_{i j}^{(N)}(\mathbf{x}),
$$

where the $\eta_{N}$ are adjustable constants. For simplicity we will again limit ourselves to only the first order effect and consider a trial function of the form

$$
\tau_{i j A}=\left\langle\tau_{i j}\right\rangle+\eta \tau_{i j}^{(1)} .
$$

To obtain the lower bound in (1) we simply set $\eta=0$ and from (14) find in view of the relation $\left\langle\beta_{i j A} \beta_{i j A}\right\rangle=\left\langle\beta_{i j A}\right\rangle\left\langle\beta_{i i_{A}}\right\rangle=0$

$$
k^{*} \geq \frac{1}{\left\langle\frac{1}{k}\right\rangle} .
$$

We seek first equations for $\psi^{(1)}$ and $\beta_{i j}^{(1)}$ similar to those obtained for $\theta^{(1)}$ and $\pi_{i j}^{(1)}[(22)$ and (23)]. We shall find these equations from (22) and (23) by noting that

$$
\begin{gathered}
\theta=\frac{\psi}{3 k}, \\
\pi_{i i}=\frac{\beta_{i i}}{2 \mu} .
\end{gathered}
$$

The right hand side of (42) may be written in terms of mean and fluctuating quantities and expanded in the form 


$$
\begin{aligned}
& \theta=\frac{1}{3\left(\langle k\rangle+k^{\prime}\right)}\left(\langle\psi\rangle+\psi^{(1)}\right) \\
& \quad=\frac{1}{3\langle k\rangle}\left[\langle\psi\rangle+\left(\psi^{(1)}-\frac{k^{\prime}}{\langle k\rangle}\langle\psi\rangle\right)+\left(-\frac{k^{\prime}}{\langle k\rangle} \psi^{(1)}+\frac{k^{\prime 2}}{\langle k\rangle^{2}}\langle\psi\rangle\right)+\cdots\right] .
\end{aligned}
$$

If (44) is substituted into (22) and only lowest order terms are retained we find after some manipulation

$$
\nabla^{2} \psi^{(1)}=\frac{4\langle\mu\rangle k^{*}\langle\theta\rangle}{\langle k\rangle(\langle\lambda)+2\langle\mu\rangle)} \nabla^{2} k^{\prime} .
$$

We initially set $\theta$ in (22) equal to $\psi / 3 k$ and to combine the remaining terms we set $\langle\psi\rangle=3\langle\theta\rangle k^{*}$.

To first order we find similarly that

$$
\pi_{i i}^{(1)}=\frac{1}{2\langle\mu\rangle} \beta_{i j}^{(1)} .
$$

Thus from (46) and (23) we have

$$
\nabla^{2} \beta_{i j}^{(1)}=\frac{2\langle\mu\rangle k^{*}\langle\theta\rangle}{\langle k\rangle(\langle\lambda\rangle+2\langle\mu\rangle)}\left[\frac{1}{3}\left(\nabla^{2} k^{\prime}\right) \delta_{i i}-\frac{\partial^{2} k^{\prime}}{\partial x_{i} \partial x_{i}}\right] .
$$

From (46) and (47) all the terms in (14) may be found exactly as in the displacement case. We present here the final result

$$
\begin{aligned}
& k^{*} \geq\left\{\left\langle\frac{1}{k}\right\rangle+\frac{2 \eta \frac{4}{3}\langle\mu\rangle}{\langle k\rangle(\langle\lambda\rangle+2\langle\mu\rangle)}\left\langle\frac{k^{\prime}}{k}\right\rangle\right. \\
&\left.+\eta^{2}\left[\frac{\frac{4}{3}\langle\mu\rangle}{\langle k\rangle(\langle\lambda\rangle+2\langle\mu\rangle)}\right]^{2}\left[\left\langle\frac{k^{\prime 2}}{k}\right\rangle-\frac{3}{8}\left\langle\frac{k^{\prime 2}}{\mu}\right\rangle+J^{\prime} \frac{9}{8}\right]\right\}^{-1}
\end{aligned}
$$

where

$$
J^{\prime}=\frac{1}{16 \pi^{2}} \int_{\xi} \int_{\eta} \frac{\partial^{2}}{\partial \xi_{i} \partial \xi_{i}} \frac{\partial^{2}}{\partial \eta_{i} \partial \eta_{i}}\left\langle\frac{k^{\prime}(\xi) k^{\prime}(\mathbf{n})}{\mu(0)}\right\rangle \frac{1}{|\xi||\mathbf{n}|} d \xi d \mathbf{n}
$$

Maximizing the right hand side with respect to $\eta$ we find

$$
k^{*} \geq\left\{\left\langle\frac{1}{k}\right\rangle-\frac{\left\langle\left(k^{\prime} / k\right)\right\rangle^{2}}{\left\langle\frac{k^{\prime 2}}{k}\right\rangle-\frac{3}{8}\left\langle\frac{k^{\prime 2}}{\mu}\right\rangle+\frac{9}{8} J^{\prime}}\right\}^{-1} .
$$

Just as in the displacement case it may be shown that (49) is at least as good a bound as that given in (1).

4. Final Result. From (39) and (49) we find that $k^{*}$ is bounded by the relation

$$
\left\{\left\langle\frac{1}{k}\right\rangle-\frac{\left(\left\langle k^{\prime} / k\right\rangle\right)^{2}}{\left\langle\frac{k^{\prime 2}}{k}\right\rangle-\frac{3}{8}\left\langle\frac{{k^{\prime 2}}_{\mu}}{\mu}\right\rangle+\frac{9}{8} J^{\prime}}\right\}^{-1} \leq k^{*} \leq\langle k\rangle-\frac{\left\langle k^{\prime 2}\right\rangle}{(\langle\lambda\rangle+2\langle\mu\rangle)+\frac{\left\langle\lambda^{\prime} k^{\prime 2}\right\rangle}{\left\langle k^{\prime 2}\right\rangle}+\frac{2 J}{\left\langle k^{\prime 2}\right\rangle}}
$$

The bounds of $k^{*}$ thus now depend upon the three point correlation functions

$$
\left\langle\mu^{\prime}(0) k^{\prime}(\xi) k^{\prime}(\mathfrak{n})\right\rangle,
$$


and

$$
\left\langle\frac{k^{\prime}(\xi) k^{\prime}(\mathfrak{n})}{\mu(0)}\right\rangle \text {. }
$$

5. Evaluation of (50) for Special Cases. Small Perturbation Case. When $\left\langle k^{\prime n}\right\rangle /\langle k\rangle^{n}$ and $\left\langle\mu^{\prime n}\right\rangle /\langle\mu\rangle^{n}$ are much less than 1 for all $n$ the bounds given in (50) coincide. The upper bound is easily evaluated since in this limit the terms $\left\langle\lambda^{\prime} k^{\prime 2}\right\rangle /\left\langle k^{\prime 2}\right\rangle$ and $2 J /\left\langle k^{\prime 2}\right\rangle$ are negligible and hence

$$
k^{*} \leq\langle k\rangle-\frac{\left\langle k^{\prime 2}\right\rangle}{\langle\lambda\rangle+2\langle\mu\rangle}
$$

The lower bound is more difficult to calculate since $J^{\prime}$ is not negligible but is approximately

$$
J^{\prime} \approx \frac{1}{16 \pi^{2}\langle\mu\rangle} \int_{\xi} \int_{\eta} \frac{\partial^{2}}{\partial \xi_{i} \partial \xi_{i}} \frac{\partial^{2}}{\partial \eta_{i}} \frac{\partial}{\partial \eta_{i}}\left\langle k^{\prime}(\xi) k^{\prime}(\mathbf{n})\right\rangle \frac{1}{|\xi||\mathbf{n}|} d \xi d \mathbf{n} .
$$

To evaluate this expression we note that $\left\langle k^{\prime}(\xi) k^{\prime}(\mathbf{n})\right\rangle=K^{\prime}(|\xi-\mathfrak{n}|)$ so that $\partial / \partial \xi_{i}=$ $-\partial / \partial \eta_{i}$ and we have

$$
J^{\prime}=\frac{1}{16 \pi^{2}\langle\mu\rangle} \int_{\xi} d \xi \frac{1}{|\xi|} \int_{\eta} \nabla_{\eta}^{2}\left[\nabla_{\xi}^{2} K^{\prime}(|\xi-\mathfrak{n}|)\right] \frac{1}{|\mathbf{n}|} d \mathbf{n}
$$

The integral over $n$ is simply $-4 \pi K^{\prime}(|\xi|)$ so that we have finally

$$
J^{\prime}=\frac{1}{\langle\mu\rangle}\left\langle k^{\prime 2}\right\rangle
$$

Substituting (54) into (50) and letting $\left\langle k^{\prime 2} / \mu\right\rangle \approx\left\langle k^{\prime 2}\right\rangle /\langle\mu\rangle$ and $\left\langle k^{\prime 2} / k\right\rangle \approx\left\langle k^{\prime 2}\right\rangle /\langle k\rangle$ yields then

$$
k^{*} \geq\langle k\rangle-\frac{\left\langle k^{\prime 2}\right\rangle}{\langle\lambda\rangle+2\langle\mu\rangle}
$$

Comparison of (51) and (54) then shows that in the limit of small perturbations

$$
k^{*}=\langle k\rangle-\frac{\left\langle k^{\prime 2}\right\rangle}{\langle\lambda\rangle+2\langle\mu\rangle}
$$

This result has been obtained by a number of investigators.

The Case of Constant Shear Modulus:

When $\mu$ is equal to a constant and only $k$ varies with position the expressions in (50) again do not depend upon the correlation functions. In this case $J=0$ since $\mu^{\prime}(\mathbf{x})=0$. The upper bound then becomes

$$
k^{*} \leq\langle k\rangle-\frac{\left\langle k^{\prime 2}\right\rangle}{\langle\lambda\rangle+2\langle\mu\rangle+\frac{\left\langle k^{\prime 3}\right\rangle}{\left\langle k^{\prime 2}\right\rangle}} .
$$

Again the lower bound is somewhat more difficult to calculate. $J^{\prime}$, however, has exactly the same value as in the small perturbation case and $\left\langle k^{\prime 2} / \mu\right\rangle=\left\langle k^{\prime 2}\right\rangle /\langle\mu\rangle$. Thus 


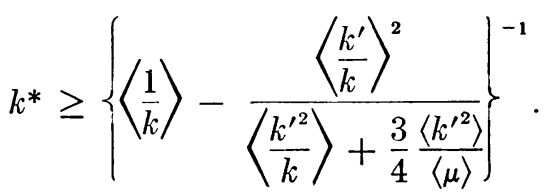

It is interesting to note that for a two phase material (i.e. the materials which have bulk modulus $k_{i}$, with volume fraction $v_{i} ; i=1,2 ; v_{1}+v_{2}=1$ ) the two bounds coincide. In this case

$$
k^{*}=\langle k\rangle-\frac{v_{1} v_{2}(\Delta k)^{2}}{\langle k\rangle+\frac{4}{3}\langle\mu\rangle+\left(v_{1}-v_{2}\right) \Delta k}
$$

where $\Delta k=k_{2}-k_{1}$. This result was obtained by Hashin and Shtrikman [2].

Two Phase Symmetric Media.

By two phase symmetric media we mean a mixture in which the statistical properties of the geometry of both phases is identical. In this particular case all three point correlation functions may be reduced to two point correlations. When this simplification is introduced into $J$ and $J^{\prime}$ they may be evaluated and the bounds given in (50) may be expressed in terms of $\Delta k=k_{2}-k_{1}$ and $\mu=\mu_{2}-\mu_{1}$.

For the upper bound the result is particularly simple since, as one might expect, the odd third order correlation function is zero. i.e.

$$
\left\langle\mu^{\prime}(0) k^{\prime}(\xi) k^{\prime}(\mathfrak{n})\right\rangle=0
$$

Thus $J=0$ and $\left\langle\lambda^{\prime} k^{\prime 2}\right\rangle=0$. The upper bound is then identical to the small perturbation solution. We have

$$
k^{*} \leq\langle k\rangle-\frac{\left\langle k^{\prime 2}\right\rangle}{\langle\lambda\rangle+2\langle\mu\rangle}=\langle k\rangle-\frac{\left\langle k^{\prime 2}\right\rangle}{\langle k\rangle+{ }^{\frac{4}{3}}\langle\mu\rangle}
$$

or

$$
k^{*} \leq\langle k\rangle-\frac{1}{4}\left[\frac{(\Delta k)^{2}}{\langle k\rangle+\frac{4}{3}\langle\mu\rangle}\right]
$$

For a symmetric medium

$$
\left\langle\frac{k^{\prime}(\xi) k^{\prime}(\mathbf{n})}{\mu(0)}\right\rangle=\frac{\langle\mu\rangle}{\mu_{1} \mu_{2}}\left\langle k^{\prime}(\xi) k^{\prime}(\mathbf{n})\right\rangle
$$

$J^{\prime}$ is now just a constant times the integral given in (53) and we find finally for the lower bound of $k^{*}$

$$
k^{*} \geq\langle k\rangle-\frac{1}{4}\left[\frac{(\Delta k)^{2}}{\left.\langle k\rangle+\frac{4}{3} \frac{\mu_{1} \mu_{2}}{\langle\mu\rangle}\right]}\right.
$$

It is interesting to compare these bounds to those obtained by Hashin and Shtrikman [2]. They find

$$
\langle k\rangle-\frac{1}{4}\left[\frac{\Delta k^{2}}{\langle k\rangle+\frac{4}{3} \mu_{1}}\right] \leq k^{*} \leq\langle k\rangle-\frac{1}{4}\left[\frac{\Delta k^{2}}{\langle k\rangle+\frac{4}{3} \mu_{2}}\right] \quad\left(\mu_{2}>\mu_{1}\right)
$$


The bounds given by (59) and (63) are better than these bounds. This is clear since $\mu_{2}>\langle\mu\rangle(\mu>0, k>0)$ and thus the upper bound is greater in (64) than in (61), and the lower bound is lower in (64) than in (63).

5. Dielectric Problem. To conclude the paper we list some results of the dielectric problem which we have not had occasion to publish elsewhere. The dielectric problem was considered in detail by Beran [3]. The bounds for the effective dielectric constant $\epsilon_{B B}^{*}$ for a symmetric two phase medium are

$$
\left[\frac{6 \alpha^{2}+6 \alpha}{\alpha^{2}+10 \alpha+1}\right] \frac{1}{\alpha^{1 / 2}} \leq \frac{\epsilon_{B E}^{*}}{\sqrt{\epsilon_{1} \epsilon_{2}}} \leq\left[\frac{2 \alpha^{2}+8 \alpha+2}{6+6 \alpha}\right] \frac{1}{\alpha^{1 / \overline{2}}}, \quad \alpha=\frac{\epsilon_{2}}{\epsilon_{1}}, \quad \epsilon_{2}>\epsilon_{1} .
$$

Bounds for this problem have also been calculated by Hashin and Shtrikman [7] and Brown [8]. They obtained the following results

$$
\left[\frac{4 \alpha+2}{5+\alpha}\right] \frac{1}{\alpha^{1 / 2}} \leq \frac{\epsilon_{H S}^{*}}{\sqrt{\epsilon_{1} \epsilon_{2}}} \leq\left[\frac{2 \alpha+4}{5 \alpha+1}\right] \alpha^{1 / 2}
$$

(Hashin and Shtrikman [7]).

$$
\left[\frac{19 \alpha^{2}+19 \alpha-2}{4 \alpha^{2}+31 \alpha+1}\right] \frac{1}{\alpha^{1 / 2}} \leq \frac{\epsilon_{B R}^{*}}{\sqrt{\epsilon_{1} \epsilon_{2}}} \leq\left[\frac{8 \alpha^{2}+26 \alpha+2}{23 \alpha^{2}+14 \alpha-1}\right] \alpha^{1 / 2}
$$

(Brown [8]).

Numerical results are plotted for the three cases in Table 1. It is seen that for this particular case of a symmetric two phase material the type of development presented

\begin{tabular}{|c|c|c|c|c|c|c|}
\hline & \multicolumn{2}{|c|}{ Hashin \& Shtrikman } & \multicolumn{2}{|c|}{ Brown } & \multicolumn{2}{|c|}{ Beran } \\
\hline & $\epsilon_{H S}^{* L} / \sqrt{\epsilon_{1} \epsilon_{2}}$ & $\epsilon_{H S}^{* u} / \sqrt{\epsilon_{1} \epsilon_{2}}$ & $\epsilon_{B R}^{* L} / \sqrt{\epsilon_{1} \epsilon_{2}}$ & $\epsilon_{B R}^{* u} / \sqrt{\epsilon_{1} \epsilon_{2}}$ & $\epsilon_{B B}^{* L} / \sqrt{\epsilon_{1} \epsilon_{2}}$ & $\epsilon_{B B}^{* u} / \sqrt{\epsilon_{1} \epsilon_{2}}$ \\
\hline$\alpha=1$ & 1 & 1 & 1 & 1 & 1 & 1 \\
\hline$\alpha=2$ & 1.01 & 1.02 & 1.01 & 1.02 & 1.02 & 1.02 \\
\hline$\alpha=5$ & .985 & 1.20 & .995 & 1.15 & 1.06 & 1.15 \\
\hline$\alpha=10$ & .889 & 1.49 & .930 & 1.37 & 1.04 & 1.35 \\
\hline$\alpha=20$ & .735 & 1.95 & .810 & 1.75 & .940 & 1.71 \\
\hline$\alpha=50$ & .520 & 2.83 & .590 & 2.58 & .720 & 2.50 \\
\hline$\alpha \rightarrow \infty$ & $4 \alpha^{-1 / 2}$ & $.4 \alpha^{1 / 2}$ & $4.75 \alpha^{-1 / 2}$ & $.348 \alpha^{1 / 2}$ & $6 \alpha^{-1 / 2}$ & $.333 \alpha^{1 / 2}$ \\
\hline
\end{tabular}
in this paper gives the best bounds.

TABLE I

\section{BIBLIOGRAPHY}

[1] Z. Hashin, Applied Mechanics Reviews, 17, 1 (1964)

[2] Z. Hashin and S. Shtrikman, Journal of Mechanics and Physics of Solids, 11, 127 (1963)

[3] M. Beran, Nuovo Cimento 38, 771 (1965) 
[4] J. Molyneux and M. Beran, Journal of Mathematics and Mechanics, 14, 337 (1965)

[5] M. Beran and J. Molyneux, Nuovo Cimento, Series X, 30, 1406 (1963)

[6] I. Sokolnikoff, Mathematical Theory of Elasticity, McGraw Hill Book Co., New York (1956)

[7] Z. Hashin and S. Shtrikman, Journal of Applied Physics, 33, 3125 (1962)

[8] W. Brown, Trans. Rheology Soc. 9, 1, p. 357 (1965) 